\title{
PHENOTYPING AND GENETIC EVALUATION OF STERILE CYTOPLASMIC MALE STERILE ANALOGUES IN SUNFLOWER (HELIANTHUS ANNUUS L.) ${ }^{1}$
}

\author{
Vikrant Tyagi*, SK Dhillon, RK BajaJ AND Shilpa Gupta \\ Department of Plant Breeding and Genetics, Punjab Agricultural University, \\ Ludhiana -141001, Punjab, India
}

Key words: CMS analogues, Genetic components, $\mathrm{D}^{2}$ analysis

\begin{abstract}
Nine new cytoplasmic male sterile (CMS) analogues on the basis of different CMS sources were developed and evaluated for morphophysiological, yield and biochemical traits. Analysis of variance indicated significant differences between CMS analogues for all the traits. The genetic components analysis indicated that selection for grain yield accompanied with high harvest index, large head size and biological yield can be effectively used for genetic improvement in sunflower. $\mathrm{D}^{2}$ analysis revealed sufficient diversity between CMS analogues which can be exploited for developing hybrids based on different cytoplasmic sources. Intra-cluster distance of 72.5 observed for the analogues present in cluster I (E002-91A, ARG-2A, DV-10A and PRUN-29A) revealed cytoplasmic dissimilarity between these sources. CMS ARG-3A was the most diverse CMS lines among all. As a result of this study the most prospective CMS analogues were designated for future use in hybrid breeding programme. The CMS analogues PRUN 29A and ARG 3 may be effectively used for developing high seed and oil yielding hybrids with desirable fatty acid composition.
\end{abstract}

\section{Introduction}

Sunflower (Helianthus annuus L., Asteraceae) is an important oilseed crop which is widely adopted and accepted for its high quality edible oil. Seed yield is an important economic character in most of the crops. This complex trait's inheritance depends upon a number of traits which are often polygenic in nature and highly affected by environmental factors. Studies on genetic divergence are important for breeding aiming to obtain hybrid cultivars, so that crosses can be made among genetically divergent lines which have contrasting and complementary traits (Luciene et al. 2010). The central component of sunflower hybrid development is cytoplasmic male sterility (CMS). So far single source of cytoplasm PET-1 has been exploited extensively for hybrid production in sunflower. There is a need to diversify the cytoplasmic base to safeguard sunflower crop from future biotic and abiotic threats, if the cytoplasm becomes susceptible. The utilization of different cytoplasmic backgrounds in hybrid development will improve general variability of the sunflower and lessen the threats of epiphytotics. Recently, several CMS backgrounds have been developed by interspecific and intraspecific crosses which resulted in more than 70 CMS sources (Serieys 2002). Since these CMS sources were identified, several experiments to estimate the influence of the cytoplasmic effect on important agronomic traits have been conducted before their introgression into commercial breeding programmes. Information on nature and magnitude of variability present in a population due to genetic and non genetic components is also an important prerequisite for systematic breeding programme. In this context the present work was undertaken to develop sterile $\mathrm{cms}$ analogues of the line NC-41B and study the effect of various cytoplasmic male sterility sources on different morphological, physiological and quality characters to exploit them in hybrid development programme.

*Author for correspondence: < vikranttyagi97@gmail.com>. ${ }^{1}$ Part of Ph.D. thesis of Vikrant Tyagi. 


\section{Materials and Methods}

The study involved germplasm of nine alloplasmic CMS lines from different cytoplasmic background viz: CMS-XA, E002-91, PKU-2 (H. annuus), ARG-2, ARG-3, ARG-6A (H. argophyllus), DV-10A (H. debilis sub spp. vestitus), PHIR-27A (H. praecox sub spp. hirtus) and PRUN-29A (H. praecox sub spp. runyonic) developed at Punjab Agricultural University, Ludhiana having a common maintainer line (NC-41B). To obtain CMS analogues all derivatives were crossed with maintainer line NC-41B followed by repeated backcrossing. The phenotypic uniformity with respect to morphological characters within these CMS analogues was obtained in $\mathrm{BC}_{6} / \mathrm{BC}_{7}$ progenies. The obtained CMS analogues were grown the field in a plot measuring $9 \times 3$ $\mathrm{m}^{2}$ during spring season 2011 in a randomized block design with three replications and evaluated for main morphological, agronomic, physiological and quality traits. The data were recorded for days to $50 \%$ flowering and days to maturity on per plot basis; for plant height, head diameter, number of leaves per plant and chlorophyll content on ten random plants in the field; for relative leaf water content, leaf water potential, leaf dry weight, leaf area, specific leaf weight and leaf area index on five random plants; and for 100 grain weight, oil content and fatty acid composition the representative sample of harvested grain, was used. Oil content was estimated using Nuclear Magnetic Resonance (NMR) and fatty acids were estimated using Gas Liquid Chromatography (GLC). The data recorded on these analogues were compared with characteristics of the line NC41B, which was used as control. The data were analyzed with the help of MVM statistical software for genetic component analysis and Indo-stat for $\mathrm{D}^{2}$ statistics of Mahalanobis (1936) followed by clustering of genotypes by Tocher's method.

\section{Results and Discussion}

The analysis of variance revealed significant differences among these CMS analogues for all traits. Since these CMS analogues have same nuclear genotype, these differences can be attributed due to differences in cytoplasmic genes/factors from different sources.

Effect of cytoplasmic sources on different characters: According to the obtained results all CMS analogues were distinct from one another with respect to most of the characters under study (Table 1). There were no significant cytoplasmic differences observed for days to flowering and maturity since the values recorded for these traits with respect to these CMS analogues did not differ significantly from NC41B.

Such uniformity for these traits between different cytoplasmic sources has also been described by previous studies (Christov 1992 and Patil et al. 2003). The cytoplasm of line CMS-XA (unknown source) had significant effect on physiological traits viz. leaf dry weight, leaf area, leaf area index and specific leaf weight per plant thereby recording maximum values for these traits (115.57 g, $2.43 \mathrm{~m}^{2}, 13.35$ and 5.10 g respectively) and differed significantly from the control NC41B which had very low values for these parameters. CMS E002-91 could be characterized as having highest oleic acid (58\%) and lowest harvest index (2.52\%) and stearic acid content (1\%). CMS PKU-2A (MUT) from wild $H$. annuus source recorded highest value for relative leaf water content (88.26) which was significantly higher than the control NC41B (78.5 \pm 2.9$)$. Amongst CMS analogues ARG 2A, ARG 3A, and ARG 6A derived from H. argophyllus source, only ARG 3A (E006) cytoplasm showed significant nuclear cytoplasmic interaction and recorded maximum value for head diameter $(24.16 \mathrm{~cm})$, biological yield $(840.00 \mathrm{~g})$, grain yield per plant $(42.67 \mathrm{~g})$ and stearic acid (5.6\%), which was substantially high than the values recorded from control NC41B for these parameters i.e. head diameter $(9.16 \pm 1.12)$, biological yield (53.3 \pm 78.8$)$, grain yield per plant (12.0 \pm 3.68$)$ and stearic acid (4.40 \pm 0.39$)$. Cytoplasm of DV-10A from H. debilis was observed to increase the number of days to maturity, though not significantly. The cytoplasmic source from H. praecox sub spp. hirtus (PHIR-27A) had significant positive 


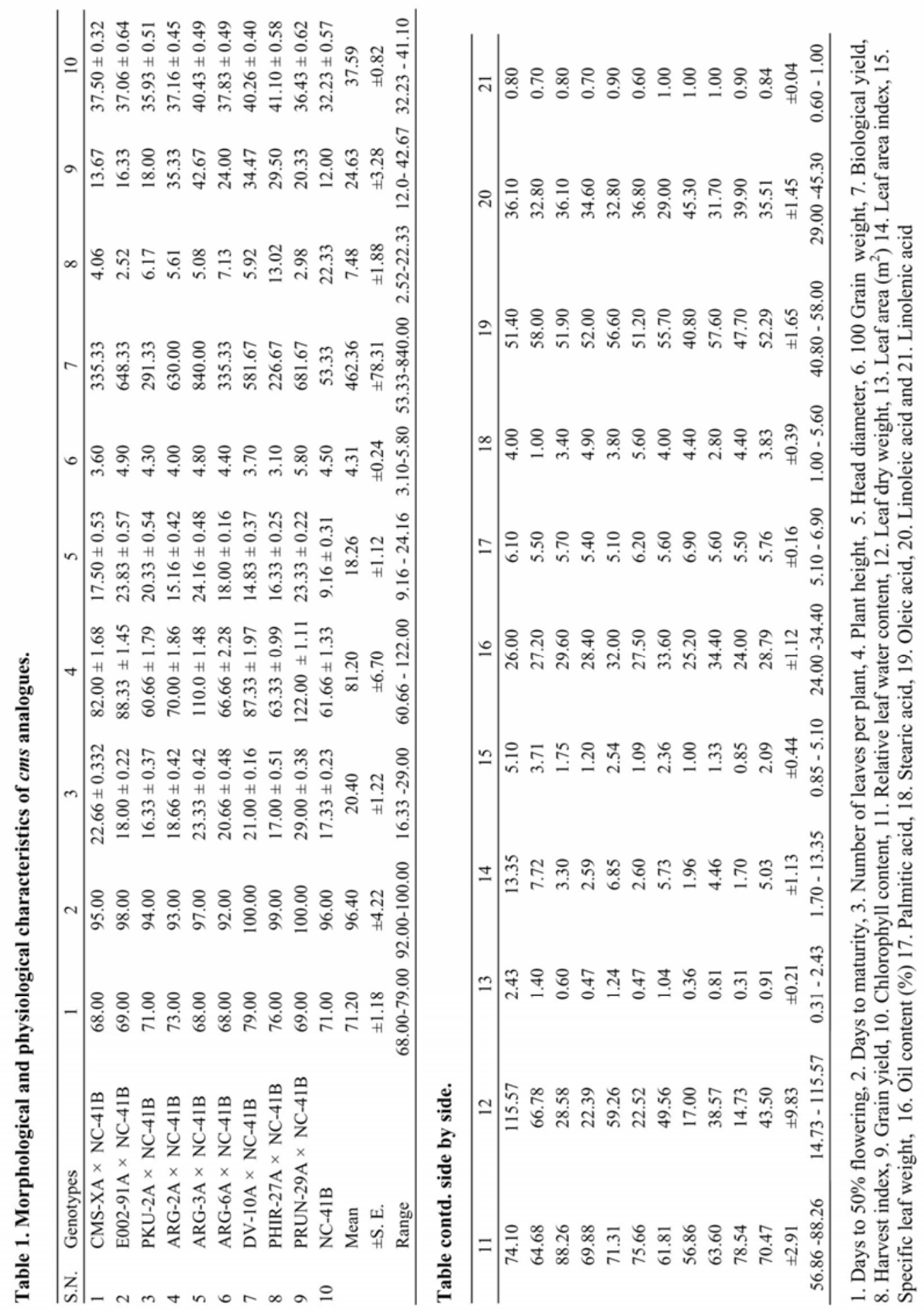


interaction for chlorophyll content, harvest index and linoleic acid concentration and recorded highest values of these three parameters (41.10, 13.02 and $45.30 \%$, respectively) which differed significantly from the control. However, (Jan 1990) has reported reduction in chlorophyll content through cytoplasmic nuclear interaction in sunflower. PRUN-29A (cytoplasm derived from

H. praecox sub ssp. runyonii gave significant positive interaction for plant height, number of leaves per plant, 100 grain weight and oil content thereby recording maximum values for these traits i.e. $122 \mathrm{~cm}, 29.00,5.80 \mathrm{~g}$ and $34.40 \%$ respectively.

CMS analogue PHIR-27A was a poor performer with respect to physiological traits viz. relative leaf water content, leaf dry weight, leaf area, leaf area index and specific leaf weight but had highest harvest index (13.02), therefore this CMS line had very good source to sink relationship.

CMS-XA derived from unknown source performed good for physiological traits but was poor in yield characters. This gives an indication that this source might not be used directly in hybrid breeding programmes as such because of its low potential for most important agronomic traits i.e. harvest index, grain yield and oil content. However, the desirable physiological traits can be transferred through back crossing in other genotypes and performance may be evaluated for further use.

The differences in the morphological, physiological and quality parameters of these CMS analogues from the control could probably be attributed to the cytoplasmic effect/nuclear interaction of these CMS sources. Previously, a number of studies have also confirmed the positive and negative influence of the cytoplasm type (Baldini et al. 1991, Matvienko 1989, Serieys 1996, 1999 and Tavoljamskiy et al. 2004) in sunflower.

Different genetic parameters i.e., heritability $\left(\mathrm{h}^{2}\right)$, phenotypic and genotypic coefficients of variance and genetic advance were computed. The phenotypic and genotypic coefficients of variation (PCV ad GCV) were highest for harvest Index $(83.90,78.71)$ followed by leaf dry weight $(65.95,63.67)$ and biological yield $(58.74,56.49)$, coupled with high heritability $(88.01$, 93.21 and 92.48, respectively) and genetic advance (18.21, 49.82 and 478.76) for these traits. High heritability coupled with high genetic advance indicates the dependence of phenotypic expression reflecting the genotypic ability to transmit genes to their off-springs. According to Johnson et al. (1955) heritability in conjunction with genetic advance would provide more reliable index of selection value. Combinations of these parameters showed that these characters are amenable for improvement through selection, particularly mass selection as described earlier (Krishnawat and Sharma 1998 and Ashok et al. 2000). Grain yield, head diameter, plant height and 100 grain weight had moderate PCV and GCV with PCV ranging from 21.77 to 43.31 and GCV ranging from 16.86 to 37.18 accompanied with moderate degree of heritability ranging from 60.01 to 80.83. However, low degree of these parameters was observed for days to $50 \%$ flowering, days to maturity, chlorophyll content, no of leaves per plant and oil content which may be attributed to the presence of both positive and negative alleles for these characters in these genotypes. Results indicate that selection for grain yield accompanied with high harvest index, large head size and biological yield from these sources can be effectively used for genetic improvement in sunflower.

Efforts were taken to group these CMS analogues based on Mahalanobis $\mathrm{D}^{2}$ statistics which indicated the presence of enough genetic diversity in the material and grouped the alloplasmic lines into four clusters. Since these CMS lines have same nuclear genotype and differ from one another with respect to cytoplasm only, so the objective was to group these lines based on their cytoplasmic differences/relatedness and to see the effect of these sources on their performance.

$\mathrm{D}^{2}$ analysis grouped the test genotypes into 4 clusters (Table 2 and Fig. 1), on the basis of aggregate differences in characters taken, with variable number of entries in each cluster 
indicating the presence of genetic diversity in the material. Clusters I and III comprised of four lines each: E002-91A, ARG-2A, DV-10A and PRUN-29A were grouped in I cluster, while PKU2A, ARG-6A, CMS-XA and PHIR-27A fell in cluster III. Remaining two clusters II and IV have one genotype each. NC-41B (control) was the only member of Cluster IV which reveals the uniqueness of this maintainer line which has cytoplasm from conventional $H$. petiolaris source. Cluster II had only one genotype i.e. ARG-3A which indicates that CMS ARG-3A is the most diverse cytoplasmic source among all. This is supported by studying Euclidean distance values of these genotypes. $\mathrm{D}^{2}$ values ranged from 47.51 to 791.22 (Table 3). Among the wild sources maximum distance was observed between ARG-3A and PHIR-27A (617.37), followed by ARG3A and PKU-2A (552.68), ARG-3A and CMS-XA (509.6), ARG-3A and ARG-6A (509.3). $\mathrm{NC}-41 \mathrm{~B}$, the genotype having cytoplasm from commercially cultivated source $H$. petiolaris was grouped in cluster IV. This had maximum Euclidean distance value of 791.22 from ARG-3A, from other genotypes this distance varied from 633.7 (PRUN-29A) to 176.9 (PHIR-27A). These results further confirmed the diversity between these CMS analogues and can be exploited for developing hybrids based on different cytoplasmic sources.

Table 2. Cluster compositions with their genotypic information.

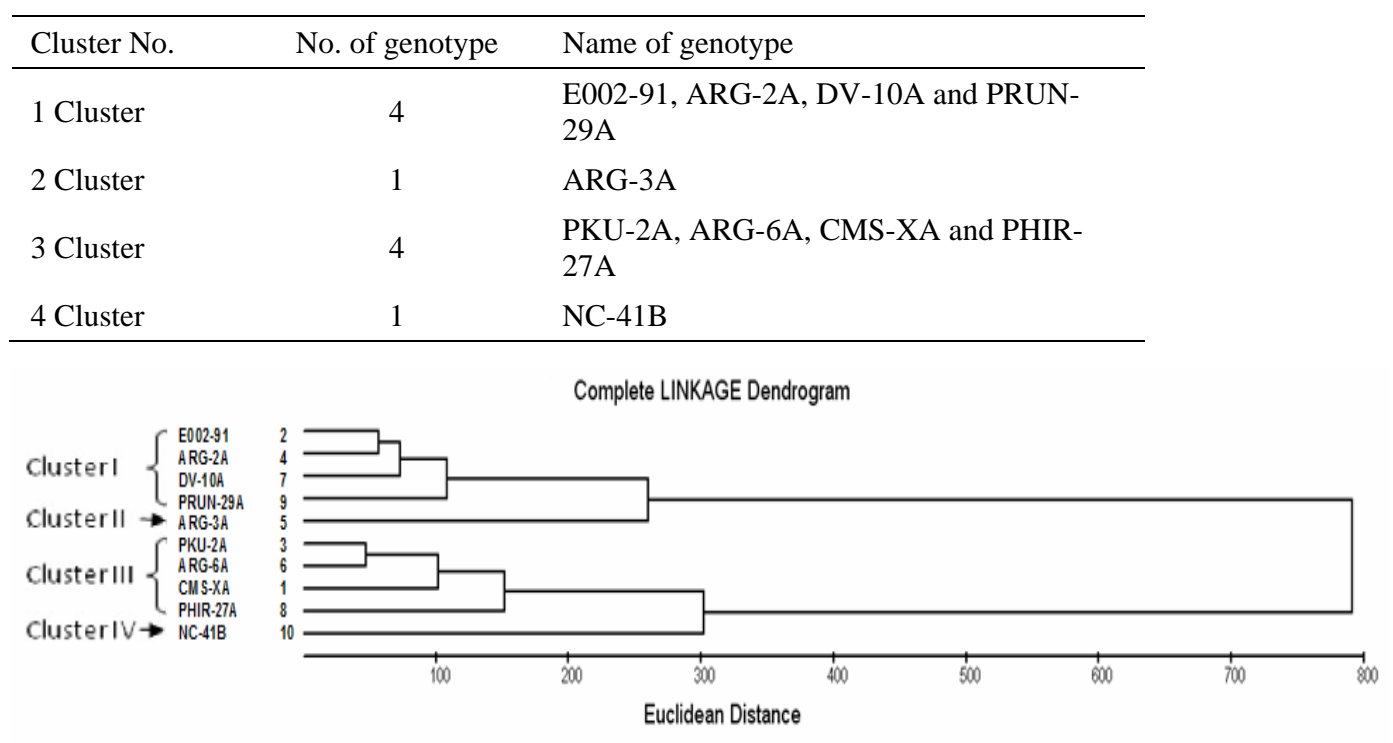

Fig. 1. Complete linkage dendogram.

Mean performance of clusters: Mean values of these clusters with respect to various characters (Table 4) indicated that cluster I comprising of four sources/lines was characterized as having maximum number of days to maturity (98 days) among all clusters. Cluster II characterized by only one line i.e. ARG-3A had lowest mean value for days to flowering (68.6), max. number of leaves per plant (22.33), highest 100 grain weight (4.56), maximum value for head diameter (24.16), high chlorophyll content (40.41) maximum value for physiological parameters i.e. leaf dry weight, leaf area, leaf area index, specific leaf weight, oil content, oleic acid, biological yield and grain yield (58.79, 1.23, 6.79, 2.63, 32.0, 56.6, 840.0 and 42.66 respectively). Cluster III again comprising of four CMS analogues had minimum number of days to maturity (95.16 days), lowest 


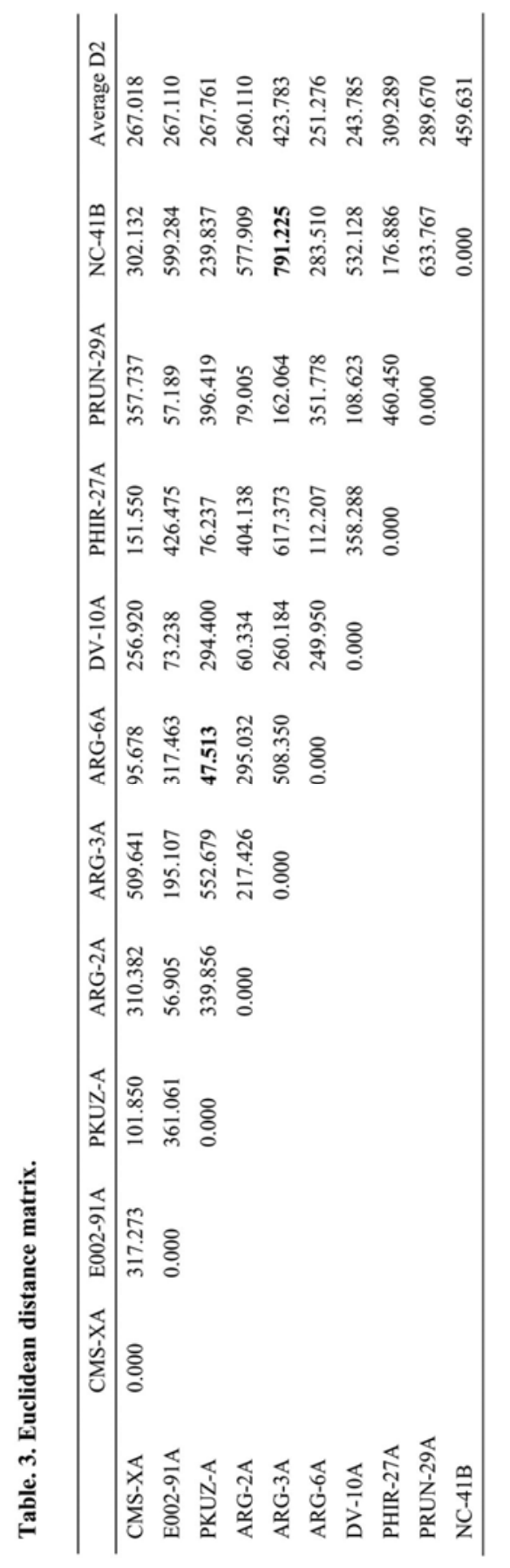


seed weight (3.85 g /100 seeds) and maximum palmitic acid composition (6.22 \%). Minimum plant height in cluster IV (68.16); max linolenic per cent acid (39.9) and high harvest index (22.32 $\%)$ was the characteristic feature of NC-41B (the common maintainer of alloplasmic lines).

Table 4. Cluster mean values.

\begin{tabular}{llllllllllll}
\hline & 1 & 2 & 3 & 4 & 5 & 6 & 7 & 8 & 9 & 10 & 11 \\
\hline 1 Cluster & 72.33 & 98.08 & 21.66 & 4.61 & 91.91 & 19.41 & 37.72 & 65.01 & 44.31 & 0.93 & 5.11 \\
2 Cluster & 68.66 & 97.00 & 22.33 & 4.86 & 110.00 & 24.16 & 40.41 & 70.32 & 58.79 & 1.23 & 6.79 \\
3 Cluster & 70.75 & 95.16 & 19.16 & 3.85 & 68.16 & 18.04 & 38.05 & 73.70 & 45.92 & 0.96 & 5.30 \\
4 Cluster & 71.00 & 96.66 & 17.33 & 4.50 & 51.66 & 9.16 & 32.26 & 78.52 & 14.70 & 0.59 & 1.70 \\
\hline
\end{tabular}

Contd. from right side.

\begin{tabular}{lllllllllll}
\hline & 12 & 13 & 14 & 15 & 16 & 17 & 18 & 19 & 20 & 21 \\
\hline 1 Cluster & 2.15 & 31.15 & 5.52 & 3.17 & 55.82 & 32.02 & 0.85 & 635.41 & 4.25 & 26.61 \\
2 Cluster & 2.63 & 32.00 & 5.10 & 3.80 & 56.60 & 32.80 & 0.90 & 840.00 & 5.07 & 42.66 \\
3 Cluster & 2.24 & 27.08 & 6.22 & 4.35 & 48.82 & 38.57 & 0.80 & 297.16 & 7.59 & 21.29 \\
4 Cluster & 0.82 & 24.00 & 5.50 & 4.40 & 47.70 & 39.90 & 0.90 & 53.33 & 22.32 & 12.00 \\
\hline
\end{tabular}

1. Days to 50\% flowering, 2. Days to maturity, 3. Number of leaves/ plant, 4. 100 grain wt. (g.), 5. Plant height $(\mathrm{cm}), 6$. Head diameter $(\mathrm{cm}), 7$. Chlorophyll cont. (\%), 8. Relative leaf water cont. (\%), 9. Leaf dry wt. (g), 10. Leaf area (m2), 11. Leaf area index, 12. Specific leaf wt./plant, 13. Oil cont. (\%), 14. Palmitic acid, 15. Stearic acid, 16. oleic acid, 17. linoleic acid, 18. Linolenic acid, 19. Biological yield, 20. Harvest index and 21. Grain yield.

Table 5. Inter and intra cluster distances.

\begin{tabular}{ccccc}
\hline & Cluster 1 & Cluster 2 & Cluster 3 & Cluster 4 \\
\hline Cluster 1 & $\mathbf{7 2 . 5 4 9}$ & 208.695 & 343.601 & 585.772 \\
Cluster 2 & & $\mathbf{0 . 0 0 0}$ & 547.011 & 791.225 \\
Cluster 3 & & $\mathbf{0 . 0 0 0}$ & 250.591 \\
Cluster 4 & & & $\mathbf{0 . 0 0 0}$ \\
\hline
\end{tabular}

The data presented in Table 5 revealed that intra-cluster distance (72.5) was observed only for the analogues present in cluster I (E002-91A, ARG-2A, DV-10A and PRUN-29A) indicating cytoplasmic dissimilarity between these lines falling within this group. Maximum inter-cluster distance of 791.22 was present for clusters II and IV followed by clusters I and IV (585.77), II \& III (547.00) and I and II (208.7).

\section{Acknowledgment}

The first author (V.T.) is thankful to the Department of Science and Technology, New Delhi, India for providing INSPIRE fellowship during this study. The Directorate of Oilseeds Research, Rajendra Nagar, Hyderabad, Andhra Pradesh, India is duly acknowledged with thanks for providing with $\mathrm{F}_{1}$ derivatives of interspecific crosses for conducting the present study.

\section{References}

Ashok S, Lakshminarayana S and Kumaresan D 2000. Variability study in Sunflower for yield and yield attributes in Sunflower. J. Oilseed Res. 17(2): 239-241.

Baldini M, Megale P and Benvenuti A 1991. Stability analysis. Cytoplasmatic effects and possible utilization on three male sterility sources in Sunflower (Helianthus). Ann. Bot. 49: 27-36. 
Christov M 1992. New sources of male sterility and opportunities for their utilization in sunflower hybrid breeding. Helia 15: 41-48.

Jan CC 1990. In search of cytoplasmic male-sterility and fertility restoration genes in wild Helianthus species. p. 3-5. In Proc. Sunflower Research Workshop, Fargo, ND. 8-9 Jan. 1990. Natl. Sun flower Assoc., Bismarck, ND.

Johnson HW, Robinson HF and Comstock RE 1955. Estimates of genetic and environmental variability in soyabeans. Agron. J. 47: 314-318.

Krishnawat BRS and Sharma SP 1998. Genetic variability in wheat under moisture stress conditions. Crop Res. 16(3): 314-317.

Luciene FC, Lázaro JC, Edward MB, Elaine B 2010. Genotypic divergence among sunflower populations. Pesq. Agropec. Trop. Goiânia. 40: 36-44.

Mahalanobis PC 1936. On the generalized distance in statistics. Proceedings of the National Institute of Science of India 12: 49-55.

Matvienko AF 1989. The influence of cms H. petiolaris type on several agronomic traits. Dissertation. Moscow. 1-162.

Patil SA, Gafoor A and Ravikumar RL 2003. Impact of cytoplasmic male sterile sources on seed yield and yield components in sunflower. Helia. 26(38): 67-72.

Serieys H 1996. Identification. study and utilization in breeding programs of new cms sources. Helia. (special issue). 19: 144-157.

Serieys H 1999. Identification. Study and utilization in breeding programs of new cms sources. Helia. 22 (special issue). 71-84.

Serieys H 2002. Report on the past activities of FAO working group "identification study and utilization in breeding program of new cms sources” for the period 1999-2001,FAO, Rome.

Tavoljanskiy NP, Chepurnaya AL, Scherstyuk SV and Tikhomirov VT 2004. Development of sunflower sterile cms analogues on the base of different cytoplasmic backgrounds. Helia. 27(40): 251-256.

(Manuscript received on 22 July, 2013; revised on 21 July, 2014) 